\title{
PSMA PET/MRI validation of MR textural analysis for detection of transition zone prostate cancer
}

\author{
ABSTRACT \\ AIM: To validate MR textural analysis (MRTA) for detection of transition zone (TZ) prostate cancer through \\ comparison to co-registered prostate specific membrane antigen (PSMA) PET-MR. \\ METHODS: Retrospective analysis was performed for 30 men who underwent simultaneous PSMA PET-MR \\ imaging for staging of prostate cancer. Thirty texture features were derived from each manually contoured \\ T2-weighted, transaxial, prostatic TZ using texture analysis software which applies a spatial band-pass filter \\ and quantifies texture through histogram analysis. Texture features of the TZ were compared to PSMA \\ expression on the corresponding PET images. The Benjamini-Hochberg correction controlled the false \\ discovery rate at $<5 \%$. \\ RESULTS: 88 T2-weighted images in 18 patients demonstrated abnormal PSMA expression within the TZ \\ on PET-MR. 123 images were PSMA negative. Based on the corrected p-value of 0.005 , significant \\ differences between the PSMA positive and negative slices were found for 16 texture parameters: Standard \\ Deviation and Mean of Positive Pixels for all spatial filters ( $p=<0.0001$ for both at all spatial scaling factor \\ $\{S S F\}$ values) and Mean Intensity following filtration for SSF 3-6mm ( $p=0.0002-0.0018)$. \\ CONCLUSION: Abnormal expression of PSMA within the TZ is associated with altered texture on T2- \\ weighted MR, providing validation of MRTA for the detection of TZ prostate cancer.
}

\section{Key Points:}

- $\quad$ Prostate transition zone (TZ) MR texture analysis may assist in prostate cancer detection.

- $\quad$ Abnormal transition zone PSMA expression correlates with altered texture on T2-weighted MR.

- $\quad$ TZ with abnormal PSMA expression demonstrates significantly reduced MI, SD and MPP.

\section{Keywords:}

1. Magnetic Resonance Imaging

2. Texture Analysis

3. Prostate

4. Cancer

5. Diagnosis

$\begin{array}{ll}\text { Abbreviations: } \\ \text { PZ } & \text { Peripheral zone of the prostate } \\ \text { TZ } & \text { Transition zone of the prostate } \\ \text { DRE } & \text { Digital rectal exam } \\ \text { TRUS } & \text { Transrectal ultrasound } \\ \text { MRTA } & \text { MRI textural analysis } \\ \text { PSMA } & \text { Prostate specific membrane antigen } \\ \text { mp-MRI } & \text { Multiparametric MRI } \\ \text { SSF } & \text { Spatial scaling factor } \\ \text { MI } & \text { Mean intensity } \\ \text { SD } & \text { standard deviation } \\ \text { MPP } & \text { Mean of positive pixels } \\ \text { AUC } & \text { Area under the receiver-operating characteristic curve } \\ \text { HASTE } & \text { Half-Fourier acquisition single-shot turbo spin echo imaging } \\ \text { GS } & \text { Gleason Score }\end{array}$




\section{Introduction:}

Prostate cancer is the most common non-cutaneous malignancy in the western world and accounts for the second highest number of deaths from cancer among males [1]. The peripheral zone (PZ) of the prostate is the most common location where prostate cancer occurs, accounting for up to $75-85 \%$ of cases. However, the transition zone (TZ) has also been identified as retrospectively containing cancer in up to $25 \%$ of radical prostatectomy specimens [2]. Current methods of prostate cancer screening include digital rectal examination (DRE) and serum prostate-specific antigen level, where diagnosis relies on histological tissue analysis through transrectal ultrasound (TRUS) guided biopsy, transperineal biopsy or prostatectomy. More recently, multi-parametric magnetic resonance imaging (mp-MRI) has been a useful tool for further investigation of patients with suspected prostate cancer as well as for biopsy planning. Although MRI is increasingly recognised as a useful adjunct to DRE and TRUS in the detection of prostate cancer, even in combination these techniques may miss TZ tumours. In the case of DRE and TRUS, the reduced sensitivity for TZ tumours is due to their anterior location. For MRI, the signal characteristics of cancer frequently overlap with other conditions occurring in the TZ, most commonly benign prostatic hypertrophy [3]. There is therefore a need for additional methods to aid the detection of prostatic cancer in the TZ.

Tumours located within the transition zone often appear as a homogenous signal mass with indistinct margins ("erased charcoal sign") and low signal intensity on the T2-weighted images that form the mainstay of the evaluation of the TZ with MRI [4]. These features can be difficult to appreciate visually but are potentially demonstrable using imaging processing techniques that assess the spatial heterogeneity of signal within tissues, known as texture analysis. Previous research has shown the potential for MR texture analysis (MRTA) to improve the detection of prostate cancer in general and in the $\mathrm{TZ}$ in particular $[5,6]$. However, these studies used histopathological examination of tissue obtained at either radical prostatectomy or trans-perineal template biopsy as the reference standard and are therefore susceptible to errors from mis-registration between histopathology and imaging findings. MRTA therefore requires further validation before translation into routine clinical practice.

Prostate Specific Membrane Antigen (PSMA) is a transmembrane protein which is found to be overexpressed on most prostate cancer cells. Through the use of a 68-Gallium radiolabelled PSMA ligand, PSMA-expressing prostate cancer can now be detected with PET-CT and PET-MR with sensitivity that is favourable compared to choline-based PET imaging techniques and CT or mp-MRI alone [7]. The accurate co-registration of PET and MRI afforded by PSMA PET-MR in particular, improves the localization of prostatic cancer in comparison to multi-parametric MRI alone [8]. PSMA PET-MR is an expensive technique with currently severely restricted access. However, the use of PSMA PET-MR as a reference standard in assessing the ability of texture analysis to detect TZ prostate cancer may help overcome the uncertainties associated with correlation between histological and imaging data. In this study, we hypothesise that PSMA PET-MR can demonstrate an association between abnormal PSMA expression within the prostatic transition zone and altered texture on T2-weighted MRI, thereby providing further validation for the application of MRTA in the detection of transition zone prostate cancer.

\section{Materials and Methods:}

This retrospective observational study formed part of a larger research programme for imaging biomarker discovery using patient data acquired as part of routine clinical care. Our local institutional review board had approved this programme and waived the requirement for individual consent.

\section{Patient Cohort:}

The cohort comprised consecutive male patients who had undergone simultaneous PSMA PET-MR including 3T multiparametric MR images of the prostate between the period of March 2015 and February 2016 for purposes of staging prostate cancer. Patients were excluded from this study if they had previously received local radiotherapy, androgen therapy, brachytherapy or prostate surgery due to the alteration in prostate tissue appearance and impact on prostatic textural analysis. In total, 30 men with a mean age (range) of 65 years $(50-81$ years) were accrued. 


\section{Image acquisition:}

All patients underwent simultaneous PET-MR with a $3 T$ magnet (Siemens, Biograph mMR PET-MR, Bayswater, Australia) following intravenous administration of $150 \mathrm{MBq}$ of $68 \mathrm{Gallium}$-labelled PSMA ligand and $5 \mathrm{mg}$ Buscopan (to minimise bowel peristalsis). Fast view and HASTE localiser (half-Fourier acquisition single-shot turbo spin echo imaging) were performed initially to allow planning of the PET and subsequent MR sequences. 45 minutes after administration of 68Gallium-labelled PSMA ligand, MRI attenuation correction and T2 sagittal/coronal/axial images were then obtained simultaneously during PET acquisition over a 15 minute period. Once PET acquisition was complete, a further $5 \mathrm{mg}$ Buscopan as well as $7.5 \mathrm{ml}$ of Gadolinium via an autoinjector were administered intravenously prior to DWI and Dynamic T1 VIBE sequences. Complete mp-MRI parameters are provided in Table 1. To enable quantitative comparison between images, T2 acquisitions utilised a standardised protocol that controlled the image gain at a predetermine level for all patients. Collected images with evidence of TZ tumour identified on T2-MR, PSMA PET and fused PSMA PET-MR are seen in Figure 1.

\section{Image Analysis:}

For all patients, the entire transition zone was manually contoured on each slice of the transaxial T2weighted image data sets containing prostatic tissue. Using TexRAD software (Feedback PLC, Cambridge, UK), 30 texture features were then derived for each whole contoured TZ by applying an increasingly course spatial band-pass filter from 2-6mm (spatial scaling factor: SSF 2-6) with subsequent quantification of the texture maps through histogram analysis. Histogram features returned for each SSF value comprised kurtosis, skewness, mean of positive pixels (MPP), entropy, mean intensity (MI) and standard deviation (SD). No image thresholding was applied prior to texture analysis.

PSMA PET images were then linked to the co-registered T2-weighted MR images and analysed by a single radiologist with specialisation in nuclear medical imaging, over 20 years of experience in PET imaging analysis and over 2 years of PSMA PET-MR analysis. Each individual PSMA PET image was then assessed for PSMA ligand uptake with positive PSMA expression defined as any region of the prostatic TZ containing focal radiotracer uptake greater than background uptake. Based on the corresponding PSMA PET image, each transaxial T2-weighted image was then classified as positive or negative for abnormal PSMA expression. Manually contoured T2-MR and corresponding texture maps are seen in Figure 2.

\section{Statistical Analysis:}

The Mann-Whitney test was used to compare each texture feature between slices in which abnormal PSMA expression was shown to be present or absent in the transition zone on the corresponding PET image. The Benjamini-Hochberg correction was then used to determine the $p$-value that would control the false discovery rate at $<5 \%$. The diagnostic performance for all texture parameters found to be significant after Benjamini-Hochberg correction was expressed as the area under the receiver-operating characteristic curve (AUC). All statistical analysis was performed using the online statistical computation site, vassarstats.net, accessed 2017.

\section{Results:}

Histopathological examination of prostatic tissue had been performed in 29 of the 30 patients; 19 by transrectal or transperineal biopsy and 20 following prostatectomy (with or without prior biopsy). The results are summarised in Table 2. Prostatic carcinoma was confirmed in 25 patients, all of whom demonstrated focally increased PSMA expression within the prostate (either the transition zone, peripheral zone or both.) The Gleason Scores (GS) of these tumours were: GS6: 2, GS7: 17, GS8: 1, GS 9:4, GS10: 1. No tumour was identified on histology in 4 cases, one of whom demonstrated focally increased radiotracer uptake in the TZ on PSMA-PET.

18 out of 30 patients were identified to have abnormal PSMA expression within the transition zone on PSMA PET-MR. Of those 18 patients, 88 individual T2-weighted images were found to have positive PSMA expression on corresponding PET images while a total of 123 images were PSMA negative. The MannWhitney test demonstrated differences between PSMA positive and negative data sets with a p-value of $<0.05$ for all SSF values for the following texture features; MI, SD, and MPP, and for kurtosis at SSF $2 \mathrm{~mm}$, skewness at SSF 4-6mm and entropy at SSF $2 \mathrm{~mm}$ (Table 3). The corrected p-value controlling the false discovery rate to less than $5 \%$ was 0.005 which resulted in significant differences between PSMA positive 
and negative images only for MPP and SD at all spatial filters, as well as MI for SSF $3-6 \mathrm{~mm}$. The most significant difference between PSMA positive and negative images was observed for MPP at SSF $=4 \mathrm{~mm}$ (Figure 3), with an AUC of 0.756 on receiver-operating characteristic analysis.

\section{Discussion:}

Our results indicate that abnormal expression of PSMA within the prostatic transition zone is associated with altered texture on T2-weighted MR. With the false discovery rate controlled at $5 \%$, significant differences between the texture analysis of T2-weighted MR images corresponding to positive and negative PSMA uptake in the TZ are seen for a range of textural features. The parameters that were statistically different (after Benjamini-Hochberg correction) were MI, SD and MPP, each parameter being reduced in the presence of PSMA expression. As these texture values are increased when image features are highlighted by the image filtration step [9], our finding of reduced values in the presence of PSMA expression is concordant with the homogenously reduced T2 signal of TZ tumours on MRI (known as the "erased charcoal" appearance) [2]. As PSMA is highly expressed in prostate cancer, this association suggests that MRTA may potentially assist in the identification of transition zone tumours which can otherwise be difficult to detect on multiparametric MR alone.

The use of abnormal PSMA expression on PET-MR as the reference standard distinguishes our study from previous reports that have assessed the potential for MRTA to aid detection of prostate cancer $[5,6]$. The simultaneous acquisition of PET and MR images ensures highly accurate co-registration of MRI features with depiction of PSMA expression on PET, thereby avoiding the potential for mis-registration that can occur when correlating imaging with histological findings. Previous studies have sought to identify tumours with Gleason grade of 7 or above as these tumours are considered clinically significant. However, PSMA expression is found in tumours with Gleason grade of less than 7 as well in higher grade tumours, and it is possible that some lower grade tumours have been detected by MRTA in our study. Nevertheless, the diagnostic performance of MRTA for detection of TZ cancer in our study is comparable to a previous MRTA study by Sidhu et al and to reported visual analysis by radiologists [2, 6, 10].

Although we performed MR at 3T, our results are concordant with those of Sidhu et al who reported textural analysis of T2 MRI performed at 1.5T in the detection of TZ prostate cancer [6] in that we also failed to find significant differences in kurtosis, entropy and skewness between TZ with and without tumour. Sidhu et al did not report results for MI, MPP and SD as they had not used a standardised MR acquisition protocol. Our adoption of a standardised MR acquisition protocol on a single imaging platform has allowed the inclusion of these texture parameters in our study, showing these parameters to be better discriminators for the presence of TZ cancer on T2-weighted images.

Our results are subject to some study limitations beyond the retrospective, observational design. Although histopathological examination of prostatic tissue had been performed in all but one of our patients, the results of tissue sampling gave limited information as to the precise location of tumour within the prostate gland and no indication as to location within any specific prostatic zone (TZ or PZ). Notwithstanding the fact all patients in our study with a tissue diagnosis of prostate cancer had positive PSMA expression within either the TZ or PZ, it remains possible that individual MR slices on T2 imaging without corresponding PSMA expression on PET could have contained non-PSMA expressing tumour. However, the probability of such occurrences is low given the reported incidence of false-negative PSMA-PET for prostate cancer of approximately $8 \%$ [11]. It is also possible that images with apparent PSMA expression may not have contained tumour either because of PSMA expression within non-malignant tissue as appeared to have occurred in subject 1 , or due to partial volume effects resulting in an apparent lesion size on PET in excess of the true tumour volume. Nonetheless, a false-detection rate is often acceptable in the diagnosis of cancer, particularly when associated with a concomitant reduction in false negative findings. 
Although important, the above limitations may be off-set by the avoidance of comparable issues associated with the co-registration of histological features after prostatectomy with prior MRI findings, as used in previous studies. As similar results have been produced by both approaches, they can be considered to provide mutual support for the validation of MRTA as a method for the detection of TZ prostatic cancer.

Our findings provide further validation for the use of MRTA as an aid to diagnosis of prostate cancer within the transition zone, with the potential for development of technology for Computer Aided Diagnosis. Altered MR texture may potentially be considered to denote an "at-risk" transition zone which could indicate biopsy rather than a watch-and-wait strategy when visual assessment of MRI is indeterminate (e.g. PIRADS 3 lesion). Alternatively, abnormal MR texture could trigger further investigation with PSMA PET-CT or PETMR.

In conclusion, this study has used PSMA PET-MR imaging to demonstrate an association between abnormal expression of PSMA within the prostatic transition zone and altered texture on T2-weighted MR. As PSMA is highly expressed in prostate cancer, this association provides validation of the the use of MRTA for the detection of transition zone prostate cancer.

\section{Compliance with ethical standards:}

\section{Guarantor:}

The scientific guarantor of this publication is Professor Kenneth Miles.

\section{Conflict of interest:}

The authors of this manuscript declare relationships with the following companies: Professor Kenneth Miles is one of the shareholders of the textural analysis software, TexRad (Feedback PLC, Cambridge, UK).

\section{Funding:}

The authors state that this work has not received any funding.

\section{Statistics and biometry:}

Professor Kenneth Miles has significant statistical expertise.

\section{Informed consent:}

Written informed consent was waived by the Institutional Review Board.

\section{Ethical approval:}

Institutional Review Board approval was obtained. 
Methodology:

- retrospective

- observational study

- performed at one institution

\section{References:}

1. Cancer Australia (2016) Prostate cancer statistics. Australian Institute of Health and Welfare, Australia. Available via https://prostatecancer.canceraustralia.gov.au/statistics. Accessed 24 July 2016 2. Akin O, Sala E, Moskowitz CS, et al (2006) Transition zone prostate cancers: features, detection, localization, and staging at endorectal MR imaging. Radiology 239:784-92

3. Hoeks CMA, Hambrock T, Yakar D et al (2013) Transition zone prostate cancer: detection and localization with 3-T multiparametric MR imaging. Radiology 266:207-217

4. Barentsz JO, Richenberg J, Clements R, et al (2012) ESUR prostate MR guidelines 2012. Eur Radiol 22:746-57

5. Khalvati F, Wong A, Haider MA (2015) Automated prostate cancer detection via comprehensive multiparametric magnetic resonance imaging texture feature models. BMC Medical Imaging 15:27

6. Sidhu HS, Benigno S, Ganeshan B, et al (2016) Textural analysis of multiparametric MRI detects transition zone prostate cancer. Eur Radiol. DOI:10.1007/s00330-016-4579-9

7. Perera M, Papa N, Christidis D, et al (2016) Sensitivity, specificity, and predictors of positive 68Gaprostate-specific membrane antigen positron emission tomography in advanced prostate cancer: a systematic review and meta-analysis. Eur Urol. DOI:10.1016/j.eururo.2016.06.021

8. Eiber M, Weirich G, Holzapfel K et al (2016) Simultaneous 68Ga-PSMA HBED-CC PET/MRI improves the localization of primary prostate cancer. Eur Urol 70:829-836

9. Miles KA, Ganeshan B, Hayball MP (2013) CT texture analysis using the filtration-histogram method: what do the measurements mean? Cancer Imaging 13:400-6

10. Oto A, Kayhan A, Jiang Y et al (2010) Prostate cancer: differentiation of central gland cancer from benign prostatic hyperplasia by using diffusion-weighted and dynamic contrast-enhanced MR imaging. Radiology 257:715-723

11. Maurer T, Gschwend JE, Rauscher I, et al (2016) Diagnostic efficacy of Gallium-PSMA positron emission tomography compared to conventional imaging in lymph node staging of 130 consecutive patients with intermediate to high risk prostate cancer. J Urol 195:1436-43

\section{Table and Figure Legend:}

Table 1.

Parameters of multi-parametric MRI sequence used during this study.

$7.5 \mathrm{ml}$ of intravenous gadolinium contrast used for contrast-enhanced MRI; DWI - diffusion weighted imaging; VIBE - volumetric interpolated breath-hold examination. 
Figure 1.

Left: Attenuation corrected PSMA PET image showing high PSMA uptake within the right sided transition zone of the prostate and physiological uptake within the urinary bladder. Middle: Corresponding transaxial T2-weighted MR image showing changes characteristic of malignancy including homogenous, hypoattenuation of the right sided prostatic transition zone ("erased charcoal sign"). Right: Fused (coregistered) transaxial PSMA PET-MR image showing high PSMA uptake within the right sided transitional zone of the prostate and physiological uptake within the urinary bladder.

Figure 2.

Unfiltered T2-weighted MR image of prostate (top left) with increasingly course spatial band-pass filtered images ( $2 \mathrm{~mm}$ - top right, $4 \mathrm{~mm}$ - bottom left and $6 \mathrm{~mm}$ - bottom right). Filtered images correspond to those from Figure 1 with highly positive PSMA uptake seen within the right sided transition zone.

Figure 3.

Box and whisker plot of all MPP values with PSMA negative results depicted on the left and PSMA positive results on the right. Box plots consist of median value, upper and lower quartiles, and range.

Table 2 .

Demonstrates the histopathological data, if available, for each patient involved in the study. Includes the presence or absence of PSMA expression within the TZ and PZ, the tissue sample analysed

(TRUS/Transperineal biopsy and/or prostatectomy), histological subtype of prostate cancer, number of positive cores on biopsy for each Gleason score, and anatomical location of prostate cancer as described on prostatectomy histopathology report. Subject number 10 represents the sole patient who has not received either a biopsy or prostatectomy to date and has been followed up with repeat PSA testing at regular intervals.

Table 3.

Median values and ranges for PSMA positive and PSMA negative, T2-weighted image slices are shown, along with their corresponding $\mathrm{P}$-values for all textural features and spatial band-pass filters. Statistically significant $\mathrm{P}$-values after application of the Mann-Whitney test and Benjamini-Hochberg procedure are shown in red. (AUC = area under receiver-operating characteristic curve.) 